\title{
Análise da influência da imagem de destinos na satisfação e fidelidade a destinações de turismo de sol e praia: Um estudo em Natal/RN
}

\author{
Analysis of tourism destination image influence on satisfaction e loyalty \\ to sun and sea tourism destinations: A study in Natal/RN
}

\begin{abstract}
Análisis de la influencia de la imagen de el destino en la satisfacción y fidelidad en los destinos turísticos de sol y playa: Un estudio realizado en Natal/RN
\end{abstract}

\author{
Márcio Marreiro das Chagas ${ }^{1}$ \\ Luciano Menezes Bezerra Sampaio ${ }^{2}$ \\ Kaline Elizama Bezerra Santos ${ }^{3}$
}

Resumo: $O$ estudo analisou a influência da imagem na satisfação e na fidelidade a destinos turísticos de Sol e Praia, investigando como caso prático Natal/RN, tendo como variáveis de controle as dimensões da qualidade. Para isto, foi realizado um estudo exploratório-descritivo, com enfoque analítico quantitativo, do tipo survey. A amostragem realizada foi probabilística, sendo o sujeito escolhido de modo aleatório simples. $\mathrm{O}$ instrumento de coleta adotado foi o questionário, aplicado junto aos turistas internacionais em visita a Natal/RN (Brasil). A coleta de dados ocorreu no Aeroporto Internacional Augusto Severo, com o total de 300 turistas entrevistados. Os resultados do estudo apontam nove dimensões da qualidade: Praias, Equipamentos Hoteleiros, Equipamentos Públicos, Restaurantes, Serviços de Transportes, Informações e Financeiro, Atrativos Turísticos, Paisagem Urbana e Natural, Diversidade de Restaurantes e Pratos, e, por último, Serviços Complementares e Acesso ao Hotel. Além disso, as hipóteses principais foram confirmadas,

\footnotetext{
1 Professor (Temporário) do Departamento de Turismo da Universidade Federal do Rio Grande do Norte (DETUR-UFRN), Natal/RN, Brasil. Professor vinculado ao Serviço Nacional de Aprendizagem Comercial do RN (SENAC/RN), Natal/RN. Doutorando em Administração pelo Programa de Pós-Graduação em Administração da UFRN (PPGA-UFRN), Natal/RN, Brasil. Mestre em Turismo pelo Programa de Pós-Graduação em Turismo da UFRN (PPGTUR-UFRN), Natal/RN, Brasil. Bacharel em Turismo pela UFRN. E-mail: marcio_marreiro@yahoo.com.br

2 Professor do Departamento de Ciências Administrativas (DEPAD-UFRN) e do Programa de Pós-Graduação em Administração da Universidade Federal do Rio Grande do Norte (PPGA-UFRN), Natal/RN, Brasil. Pós-doutorado na University of Illinois, Chicago, Estados Unidos da América. Doutorado em Economia pela Universidade Federal de Pernambuco (UFPE), Recife/PE, Brasil, com Estágio doutoral em Paris 1 - Sorbonne, Paris, França. Mestrado em Engenharia de Produção Universidade Federal de Pernambuco (UFPE), Recife/PE, Brasil. Graduação em Engenharia Civil na Universidade Federal de Pernambuco (UFPE), Recife/PE, Brasil. E-mail: luciano.sampaio@pq.cnpq.br

${ }^{3}$ Graduada em Turismo pela Universidade Federal do Rio Grande do Norte (UFRN). Email: kalinelizama@yahoo.com.br
} 
ou seja, a imagem de destinos afeta direta e fortemente o processo de satisfação e fidelização de turistas. Além disso, a qualidade exerce também influência significativa na satisfação e fidelização.

Palavras-Chave: Imagem de destinos; Qualidade; Satisfação; Fidelidade; Modelos matemáticos.

Abstract: This paper analysis tourism destination image influences on satisfaction and loyalty to Sun and Sea tourism destinations, investigating Natal/RN as a case. Quality dimensions were control variables. Therefore, it was conducted an exploratory and descriptive research with quantitative analytical approach. Data collection was performed by a questionnaire addressed to International tourists at the Augusto Severo International Airport. The composition was a simple random sampling by reaching the final number of 300 international tourists interviewed. Among the main results, it was noted that nine are the dimensions of perceived Quality of Sun and Sea segment, it means, Beaches, Public Equipment, Restaurants, Transportation, Information and Finance Services, Hotel Equipment, Diversity of Restaurants and Food, Complementary Services and Access to the hotel and Tourism Attractions, Urban and Natural Scenery. From the results, it was concluded that tourism destination image influences satisfaction and loyalty. Moreover, quality seems to be other important dimension for the satisfaction and loyalty process.

Keywords: Tourism destination image; Quality; Satisfaction; Loyalty; Mathematic models.

Resumén: Este artículo analizó la influencia de la imagen en la satisfacción y fidelidad a los destinos turísticos de Sol y Playa, las dimensiones de la calidad fueran las variables de control. Para esto, se realizó un estudio exploratorio descriptivo, de tipo Survey, con un enfoque analítico cuantitativo. La recolección de datos se realizó a través de una entrevista aplicada junto a los turistas internacionales en el Aeropuerto Internacional Augusto Severo en la Ciudad de Natal. La composición de la muestra fue aleatoria simple; y contó con 300 turistas entrevistados. Entre los principales resultados se observó que son nueve las dimensiones percibidas de la calidad en los destinos de sol y playa: Playas, Equipamientos del Hotel, Alimentos y Bebidas, Servicios de Transporte, Información y Financiero, Atractivos Turísticos, Paisaje Urbana y Natural, Diversidad de los Restaurantes y los Platos, y por último, Servicios complementarios y el Acceso al Hotel. Además, se concluye que la imagen de destinos afecta directamente y fuertemente el proceso de satisfacción y fidelidad de los turistas. La calidad también tiene una influencia significativa en la satisfacción y fidelidad del cliente.

Palabras clave: Imagen de destinos; Calidad; Satisfacción; Fidelidad; Modelos matemáticos

\section{INTRODUÇÃO}

A atividade turística a cada ano ratifica sua posição hegemônica entre os principais setores econômicos mundiais, como afirmam seus principais órgãos em escala internacional e nacional tais como a WTTC (2007), OMT (2005) e o MTUR (2007). Essa acentuada movimentação financeira promovida pelo turismo, entre outros impactos causados por ele, despertam em inúmeras localidades o anseio no desenvolvimento desta atividade econômica com vistas a mitigar seus problemas socioeconômicos através do estímulo ao desenvolvimento local (Mowforth \& Munt, 2006; Andereck et al, 2005).

Nesse sentido, é possível observar nos últimos anos um crescimento geométrico no 
número de localidades que passaram a promover o turismo em seu território aumentando a competitividade internacional (Ritchie \& Crounch, 2003; Chen \& Tsai, 2006; Yüksel, 2001; Valls, 1996). O acirramento da competitividade é observado não apenas na escala de destinos, como também de empreendimentos turísticos que concorrem agora em escala global por um público, por um lado, crescente e com grandes possibilidades de expansão e, por outro, cada vez mais escasso em razão do elevado nível de concorrência e competitividade encontradas na área.

Diante deste cenário, é que se potencializa a importância do desenvolvimento de estratégias que estimulem o potencial competitivo de destinos e empreendimentos turísticos (Rimmigton \& Kozak, 2000; Valls, 1996; Ritchie \& Crounch, 2003; Kozak, 2001). Para tanto, faz-se necessário informações cada vez mais precisas e refinadas sobre as nuances dos mercados e do comportamento do consumidor de turismo com vistas a pautar as decisões estratégicas dos gestores dos destinos e das próprias organizações privadas (Assael, 1999; Valls, 1996). Em outras palavras, o estudo do comportamento do consumidor se torna condição sine qua non para o pleno desenvolvimento das potencialidades do setor perante esse acentuado mercado competitivo global.

Dentre os principais tópicos relacionados a essa área de estudo, observa-se na literatura a concentração em aspectos estratégicos para destinações e empresas, com importância reconhecida tanto por acadêmicos como por profissionais da área, tais como os fatores que influenciam a escolha da destinação de férias pelo consumidor de turismo (Correia \& Pimpão, 2005; Sirakaya \& Woodside, 2005; Nicolau \& Más, 2006). Assim como, os constructos qualidade, satisfação e fidelidade que também apresentam elevado número de estudos tanto na área de serviços (de modo amplo), como, por exemplo, Oliver (1993), Parasuraman, Zeithaml \& Berry (1985; 1988), Cronin \& Taylor (1994), Johnson (2001) entre outros, como também, especificamente, para a área de turismo, principalmente no que diz respeito a sua relação causal (Chi \& Qu, 2008; Bosque \& Martín, 2008; Zabkar et al, 2009; Tsang \& Qu, 2000; Yoon \& Uysal, 2005; Correia \& Pimpão, 2005; Rimmigton \& Kozak, 2000).

Em outra linha, complementar, estudos sobre o constructo imagem são bastante recorrentes na área de comportamento do consumidor e marketing como afirmam Echtner \& Ritchie (1991), Gallarza, Gil \& Calderón (2002), Baloglu \& McCleary (1999), Beerli \& Martin (2004a), Beerli \& Martin (2004b), seja por sua relevância destacada como variável influenciadora no processo de seleção de destinos (Bosque \& Martín, 2008; Chen \& Tsai, 2006; Chi \& Qu, 2008; Echtner \& Ritchie, 1991; Pike, 2002; Gallarza, Gil \& Calderón, 2002), ou como uma das principais influenciadoras da satisfação e fidelidade a eles (Chi \& Qu, 2008; Gutiérrez, 2005; Pike, 2002).

Faz-se mister ressaltar, no entanto, que embora a importância teórica e prática de cada constructo, como também de sua relações, serem amplamente reconhecidas como primordiais para ações estratégias em qualquer setor, inclusive no turismo, a literatura da área ainda não apresenta um corpo teórico consolidado sobre a relação causal entre imagem, qualidade, satisfação e fidelidade a destinações de férias. As pesquisas têm sido desenvolvidas, grosso modo, 
a partir de adaptações de outras áreas, mas, não constituindo, ao longo do tempo, um corpo teórico próprio robusto como é necessário para uma atividade tão específica e estratégica como a turística (Bedia, Fernández \& López, 2007; Yilmaz, 2009; Juwaheer, 2004; Akbaba, 2006; AlbaceteSáez, Fuentes-Fuentes \& Lloréns-Montes, 2007).

Em resumo, se pode afirmar que a despeito do reconhecimento da importância de tais constructos e relações a área de turismo carece de estudos específicos, mais precisamente no que diz respeito ao desenvolvimento de modelos e instrumentos próprios (Bedia, Fernández \& López, 2007; Yilmaz, 2009; Juwaheer, 2004; Akbaba, 2006; Albacete-Sáez, Fuentes-Fuentes \& LlorénsMontes, 2007), como também da análise da relação causal entre os constructos mencionados (Chi \& Qu, 2008; Bosque \& Martín, 2008; Chen \& Tsai, 2006; Zabkar, Brencic \& Dmitrovic, 2009; Yoon \& Uysal, 2005; Correia \& Pimpão, 2005).

Dessa maneira, esse estudo tenta contribuir para a redução destas lacunas. O objetivo principal é analisar a relação causal entre Imagem de Destinos, Qualidade de Serviços, Satisfação e Fidelidade. Especificamente, foi analisado o impacto da imagem na satisfação e fidelidade, tendo as dimensões da qualidade de serviços como variáveis de controle. Além disso, se pretendeu examinar quais são as dimensões da qualidade de serviços em destinos turísticos de Sol e Praia e como elas se relacionam com os demais constructos. Para tanto, é sugerido um instrumento específico para a avaliação da qualidade de serviços em destinações de Sol e Praia. O estudo foi desenvolvido na Cidade do Natal com o público internacional. A seguir será apresentado o marco teórico, constituído por alguns dos principais modelos tomados como base para o desenvolvimento da investigação.

\section{MOdelos de AVALIAÇÃo dA RELAÇÃo ENTRE IMAgEM, QUALIDADE, SATISFAÇÃo E FIDELIDADE A DESTINOS TURÍSTICOS}

\subsection{Modelo de Baker \& Crompton (2000)}

O estudo de Baker \& Crompton (2000), procura analisar a qualidade e a satisfação como antecedentes da fidelidade. Para tanto, discutem e testam empiricamente qual a abordagem mais adequada para a avaliação da qualidade, se através da Teoria da Desconfirmação das Expectativas de Parasuraman et al (1985; 1988) e Oliver (1980; 1993). Ou, por outro lado, se é através da concepção da avaliação de desempenho adotada Cronin \& Taylor (1994), entre outros. Os autores testaram duas hipóteses principais, a primeira afirma que a qualidade percebida possui maior influência total na explicação do comportamento futuro (fidelidade) que a satisfação. Enquanto que a segunda afirma que o modelo baseado no desempenho possui maior adequabilidade para explicação do comportamento futuro (fidelidade) que o modelo de desconfirmação da expectativa.

Dentre os principais resultados, observou-se no teste da abordagem do desempenho que a 
qualidade exerce influência direta e significativa na satisfação. Assim como, a qualidade e a satisfação exercem influência direta e significativa junto à fidelidade. Além disso, também se conclui que a satisfação não media completamente a influência da qualidade na fidelidade, ou seja, a qualidade exerce influência direta e indireta através da satisfação. A adequabilidade do modelo baseado na performance (desempenho) foi superior ao modelo baseado na Teoria da Desconfirmação das Expectativas, como é observado em Hui, Wan \& Ho (2007), Armstrong, Mok, Go \& Chan (1997) e Yilmaz (2009). Desse modo, foram confirmadas as duas hipóteses propostas.

A maior adequabilidade do modelo de perfomance (desempenho) proposto por Cronin \& Taylor (1994), segundo Baker \& Crompton (2000), se deve ao fato da maior facilidade em avaliar e interferir nestes fatores. Afinal, levar em consideração apenas o desempenho é ponderar apenas aspectos passíveis de gerenciamento interno.

\subsection{Modelo de Um, Chon \& Ro (2006)}

O estudo desenvolvido por Um, Chon \& Ro (2006) objetivou analisar os constructos Atratividade Percebida, Qualidade Percebida dos Serviços, Valor e Satisfação como antecedentes da intenção de visitar o destino novamente. Como pode ser visto, acrescenta, se comparado ao modelo de Baker \& Crompton (2000), a dimensão valor na análise.

Nesse modelo, a relação entre Atratividade Percebida e Satisfação foi a que apresentou o maior coeficiente de correlação, ao passo que a relação menos significativa foi encontrada entre a qualidade percebida e a intenção de retornar ao destino, diferentemente ao encontrado por Baker \& Crompton (2000), onde ao se somar a influência direta e indireta constatou-se que a qualidade apresentava a maior influência na fidelidade ao destino. Ainda de acordo com a investigação de Um, Chon \& Ro (2006), a Atratividade Percebida foi a variável com maior influência junto a intenção de retorno, inclusive, maior que a própria satisfação. Esta última foi influenciada fortemente pelas variáveis Atratividade Percebida, Qualidade e Valor, sendo a primeira citada (Atratividade Percebida) a mais influente também na explicação da satisfação. A qualidade, por um lado, influenciou a satisfação, mas, por outro, não foi encontrada influência na intenção de retorno. A dimensão valor, diferentemente da qualidade, influenciou tanto satisfação quanto a intenção de retorno.

Desta pesquisa, faz-se mister ressalta que a Atratividade Percebida demonstrou maior importância que a satisfação na explicação da intenção de retornar. Em outras palavras, observouse que os fatores que atraem o turista ao destino são mais importantes para fidelização deles do que as dimensões responsáveis por sua satisfação com a destinação. Outra conclusão interessante foi que a distância do destino de férias é um dos fatores primordiais para a intenção de revisitar, maior que o próprio fator cultural no processo. 


\subsection{Modelo de Chi e Qu (2008)}

O modelo desenvolvido por Chi \& Qu (2008), acrescenta à discussão a dimensão imagem de destinos. Dito de outro modo, esses autores procuram explicar a fidelidade em função da satisfação com os atributos, utilizando-se direcionadores similares aos de qualidade usados por Baker \& Crompton (2000) entre outros, da satisfação global e da imagem do destino. Esta última variável, de acordo com as suposições de Chi \& Qu (2008), possui grande relevância na explicação da satisfação com os atributos, da satisfação global e da própria fidelização. Este modelo inclui na análise uma importante dimensão, a imagem de destinos, que ainda não tinha sido considerada nos modelos anteriormente citados. Embora seja de amplo conhecimento a influência exercida pela imagem na satisfação e fidelidade, como observado em Gutiérrez (2005) e Pike (2002).

Do estudo de Chi \& Qu (2008), conclui-se que a imagem influencia fortemente a satisfação com os atributos e a satisfação global, enquanto que afeta a fidelização apenas por intermédio da satisfação global. Ou seja, a imagem não apresentou significância na explicação direta da fidelização, como encontrado em Bosque \& Martin (2008), demonstra influência, apenas, através da satisfação global. Esta, por sua vez, é influenciada também pela satisfação com os atributos, e exerce poder explicativo junto à fidelização ao destino de férias. Do estudo, não se pode afirmar que a satisfação global media completamente a relação entre satisfação com os atributos e fidelidade ao local de férias, é possível considerar tal relação mediada apenas parcialmente pela satisfação global. Desta investigação, conclui-se a relevância estratégica dos investimentos em imagem de destinos para a elevação da avaliação da satisfação com relação à satisfação dos atributos e global, assim como para o aumento das taxas de retorno de turistas a destinação de férias, embora mediada pela satisfação.

\subsection{Modelo de Wang, Zhang, Gu \& Zhen (2009)}

O estudo objetivou realizar a modelagem causal entre os fatores que afetam a satisfação junto aos fatores por ela afetados. Em outras palavras, procurou analisar quais fatores influenciam a satisfação, apontando como antecedentes dela a percepção de valor, a qualidade percebida, as expectativas e a imagem. E a satisfação, por sua vez, foi testada como influenciadora das reclamações e da fidelidade ao destino.

De acordo com Wang, Zhang, Gu \& Zhen (2009), as expectativas afetam positivamente a satisfação e qualidade percebida, mas não a dimensão valor. Já a imagem influencia positivamente a satisfação, como observado em Chi \& Qu (2008), como também a qualidade percebida, a percepção de valor e as expectativas do turista. A percepção de valor é influenciada positivamente pela qualidade percebida, ao passo que afeta, como esta última também (qualidade percebida), a satisfação. Já as reclamações dos turistas estão negativamente relacionadas com a satisfação e a fidelidade ao destino de férias. Por fim, observou-se a relação direta e positiva entre satisfação e 
fidelidade, demonstrada em outros estudos como Zabkar, Brencic \& Dmitrovic (2009), Hui, Wan \& Ho (2007), Chi \& Qu (2007), Chen \& Tsai (2006), Armstrong, Mok, Go \& Chan (1997), Tsang \& Qu (2000), Bosque \& Martín (2008), Yilmaz (2009) entre outros. O estudo demonstrou que a maior influência na satisfação tem origem na imagem de destinos (Diretos e indiretos juntos), e da qualidade quando considerado apenas o efeito direto.

\section{METODOLOGIA DA PESQUISA}

O estudo desenvolvido é exploratório-descritivo no que diz respeito aos seus objetivos. 0 enfoque analítico mais adequado para o cumprimento de sua finalidade foi o quantitativo, sendo a investigação caracterizada como do tipo Survey. O local selecionado para a pesquisa foi a Cidade do Natal, estudo de caso deste município, um dos principais destinos turísticos internacionais do Nordeste do Brasil, como também avaliado como destino indutor do turismo pelo próprio Ministério do Turismo (2007). Já o público-alvo foi o de turistas internacionais, ou seja, aqueles provenientes de outros países que não o Brasil, com objetivo de lazer, sem remuneração e com estadia inferior a 1 ano e superior a 24h (OMT, 2005). O processo amostral foi probabilístico, sendo a escolha do sujeito realizada de modo aleatório simples. O tamanho da amostra foi definido a partir da fórmula matemática, para população infinita, recomendada pela Organização Mundial de Turismo (2005) para pesquisas de mercado deste tipo.

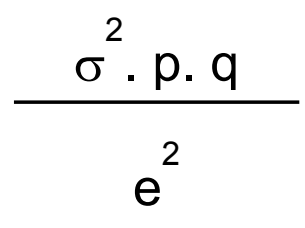

Fonte: OMT (2005)

Nesta fórmula matemática, " $n$ " é o tamanho da amostra, " $\sigma^{2}$ " é o nível de confiança escolhido, em termos de desvio padrão, sendo nesta pesquisa estipulado em $95 \%$ ou dois (02) desvios padrão; "p" é a percentagem com a qual o fenômeno se verifica, 50\% para estudos deste tipo como recomendam Hair Jr. et al (2006); e " $q$ " é a percentagem complementar (100 - p). 0 " $\mathrm{e}^{2 \text { " }}$ representa o erro máximo permitido que, para fins desta investigação, foi estimado em $5 \%$ como preconizado por Corrar et al (2007). Nesse sentido, foi obtido o número de 300 entrevistados como amostra final da pesquisa.

O processo de coleta de dados ocorreu no período compreendido entre 20 de Abril e 16 de Maio de 2009, a partir de um questionário estruturado aplicado aos turistas internacionais no Aeroporto Internacional Augusto Severo, no momento de espera pelo vôo de retorno aos seus países de origem. Os questionários foram aplicados nos idiomas inglês, espanhol, italiano e português de Portugal.

O modelo de análise adotado para avaliar a relação causal entre Imagem de Destinos, 
Qualidade dos Serviços, Satisfação e Fidelidade aos destinos turísticos de Sol e Praia foi baseado no modelo de Chi \& Qu (2008), onde se mantiveram os constructos enquanto que os direcionadores foram adaptados para destinos turísticos de Sol e Praia. A adaptação do modelo pode ser observada na Figura 01.

Figura 01 - Adaptado do modelo de Chi \& Qu (2008).

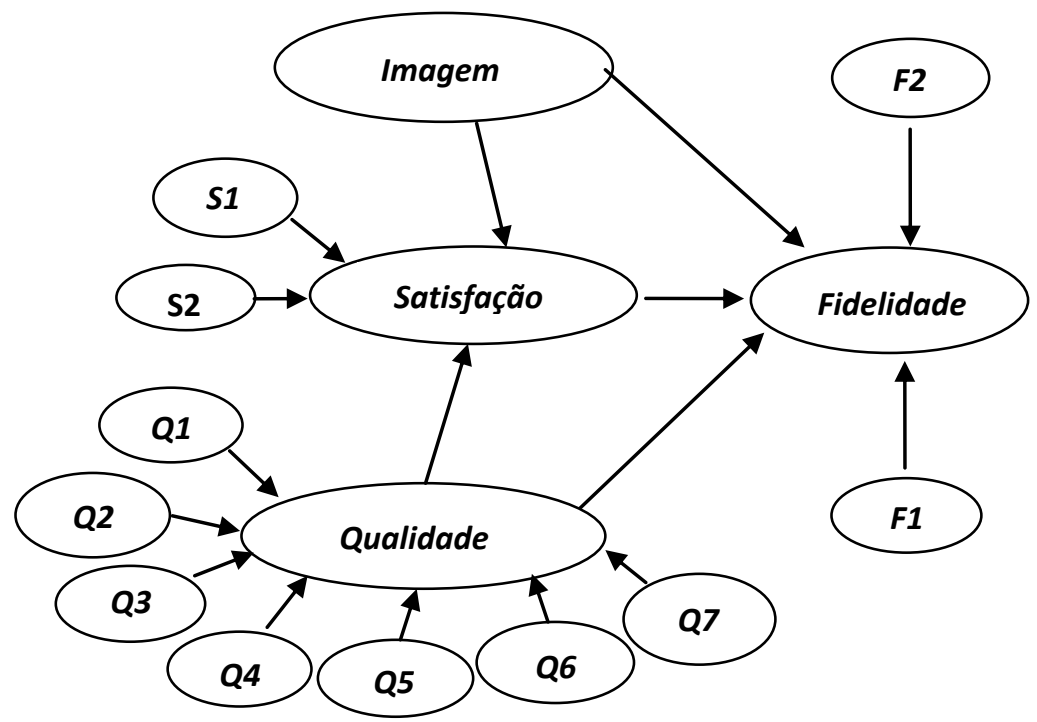

Fonte: Elaboração própria.

Do exposto na Figura 01, observa-se que a Imagem de Destinos foi operacionalizada a partir de variável única. O constructo Qualidade dos Serviços, por sua vez, é função de sete dimensões principais, a saber: (Q1) Qualidade dos Meios de Hospedagens, (Q2) Qualidade em Alimentos e Bebidas, (Q3) Praias, (Q4) Qualidade dos Equipamentos de Entretenimento, (Q5) Qualidade dos Atrativos Turísticos, (Q6) Qualidade da Infraestrutura Pública/Geral e (Q7) Qualidade dos Serviços de Transportes. Cada dimensão apresentada é constituída por um subconjunto de variáveis que podem ser observada na Tabela 02. Em síntese, é possível representar a relação da seguinte maneira: Qualidade de Serviços de Destinos de Sol e Praia = Q1 + Q2 + Q3 + Q4 + Q5 + Q6 + Q7. As dimensões e variáveis selecionadas para composição da Qualidade de Serviços foram baseadas em Chi \& Qu (2008), Hui, Wan \& Ho, (2007), Chen \& Tsai (2006), Rimmigton \& Kozak (2000), Andriotis, Agiomirgianakis \& Mihiotis, (2008), Yüksel (2001), Kozak (2000) entre outros.

A Satisfação com o Destino (S3), é função de dois direcionadores, ou seja, a Satisfação Global (S1) e a Satisfação com o Custo-Benefício do Destino (S2). Representado de outra maneira, tem-se Satisfação $(S 3)=S 1+S 2$. Estas variáveis foram extraídas de estudos tais como Yilmaz (2009), Hui, Wan \& Ho, (2007), Juwaheer (2004) Akbaba (2006) Albacete-Sáez, Fuentes-Fuentes \& Lloréns-Montes (2007) e Chi \& Qu (2008) entre outros. 
Por último, a Fidelidade (F3) também é função de dois direcionadores, sendo eles a Intenção de Retornar ao Destino (F1) e a Propensão a Recomendar o Destino (F2). A relação pode ser representada da seguinte maneira: F3 = F1 + F2. Os direcionadores foram baseados em Yilmaz (2009), Juwaheer (2004) Akbaba (2006) Albacete-Sáez, Fuentes-Fuentes \& Lloréns-Montes (2007), Chi \& Qu (2008), Hui, Wan \& Ho, (2007), Bosque \& San Martin (2008), Chen \& Tsai (2006) entre outros.

O instrumento de coleta de dados foi do tipo questionário, baseado em outros instrumentos amplamente reconhecidos na literatura internacional de serviços, tais como o SERVQUAL de Parasuraman, Zeithaml \& Berry $(1985 ;$ 1988) e SERVPERF de Cronin \& Taylor (1994). Cabe ressaltar, que foi utilizada a concepção teórica de avaliação apenas de desempenho, sendo, de tal modo, condizente com a concepção do SERVPERF. As dimensões e variáveis foram todas adaptadas para destinos turísticos de Sol e Praia, baseados nos estudos supramencionados, não constituindo, desse modo, os instrumentos SERVQUAL e SERVPERF originais. O questionário foi baseado na escala Likert de dez (10) pontos, sendo [01] o valor referente à menor avaliação de cada variável e [10] a maior, existindo, ainda, a opção [99] para aqueles que não tinham experimentado o serviço ou desenvolvido opinião sobre ele.

Por fim, para fins de análise dos dados, foi utilizado o software Statistical Package for the Social Science SPSS (17.0). O processo foi desenvolvido a partir de Análises Estatísticas Descritivas, em um primeiro momento, seguidas de Análise Multivariadas de Dados, especificamente, a Análise Fatorial Exploratória (AFE) e a Análise de Regressão Linear Múltipla (RLM), cujos parâmetros e testes estatísticos necessários para a validação de seus resultados são apresentados no tópico seguinte de discussão dos resultados.

\section{DISCUSSÃO DOS RESULTADOS}

\subsection{Validação da Amostra}

A amostra do estudo foi constituída por turistas internacionais como citado na metodologia. Dentre os entrevistados, $26,3 \%$ eram de nacionalidade portuguesa, a maioria, seguida pela italiana $(22,9 \%)$, holandesa $(14,5 \%)$, inglesa $(13,1 \%)$ e espanhola $(10,4 \%)$. Esta estratificação é coerente com as pesquisas de demanda turística internacional elaborada pela Secretaria de Estado do Turismo (SETUR/RN 2007), embora o público britânico seja menor que o espanhol nos relatórios da secretaria. As demais nacionalidades obtiveram menor representatividade, totalizando $13,8 \%$ de entrevistados. O gênero feminino obteve leve predominância sobre o masculino, $51 \%$ e $49 \%$, respectivamente.

O nível de escolaridade afirmado por $54,6 \%$ dos entrevistados foi o superior, concluído ou em processo de conclusão. Seguidos por $23,6 \%$ com nível médio (completo ou incompleto); e $16,9 \%$ afirmaram ser pós-graduados, apenas $4,9 \%$ do público afirmaram ter o equivalente ao 
ensino fundamental. No que diz respeito à faixa etária, mais da metade encontra-se com idade entre 26 e 50 anos, sendo 27,2\% entre 26 e 35 e 32,2\% entre 36 e 50 anos, o que poderia encontrar justificativa no fato desta ser a idade em que se atinge certa estabilidade financeira. Quando se trata da forma de vinda a Natal, 42,7\% afirmaram ter vindo com a família, seguidos de $39,8 \%$ que viajaram acompanhados pelos amigos.

Com relação à frequência de visitas a Cidade do Natal, observou-se que $34,2 \%$ dos visitantes quase nunca vêm à cidade, seguidos de $29,8 \%$ que nunca tinham vindo à cidade e $28,4 \%$ que às vezes fazem uma visita ao destino. A Tabela 01 apresenta o resumo do perfil dos entrevistados.

Tabela 01 - Perfil da amostra

(continuação)

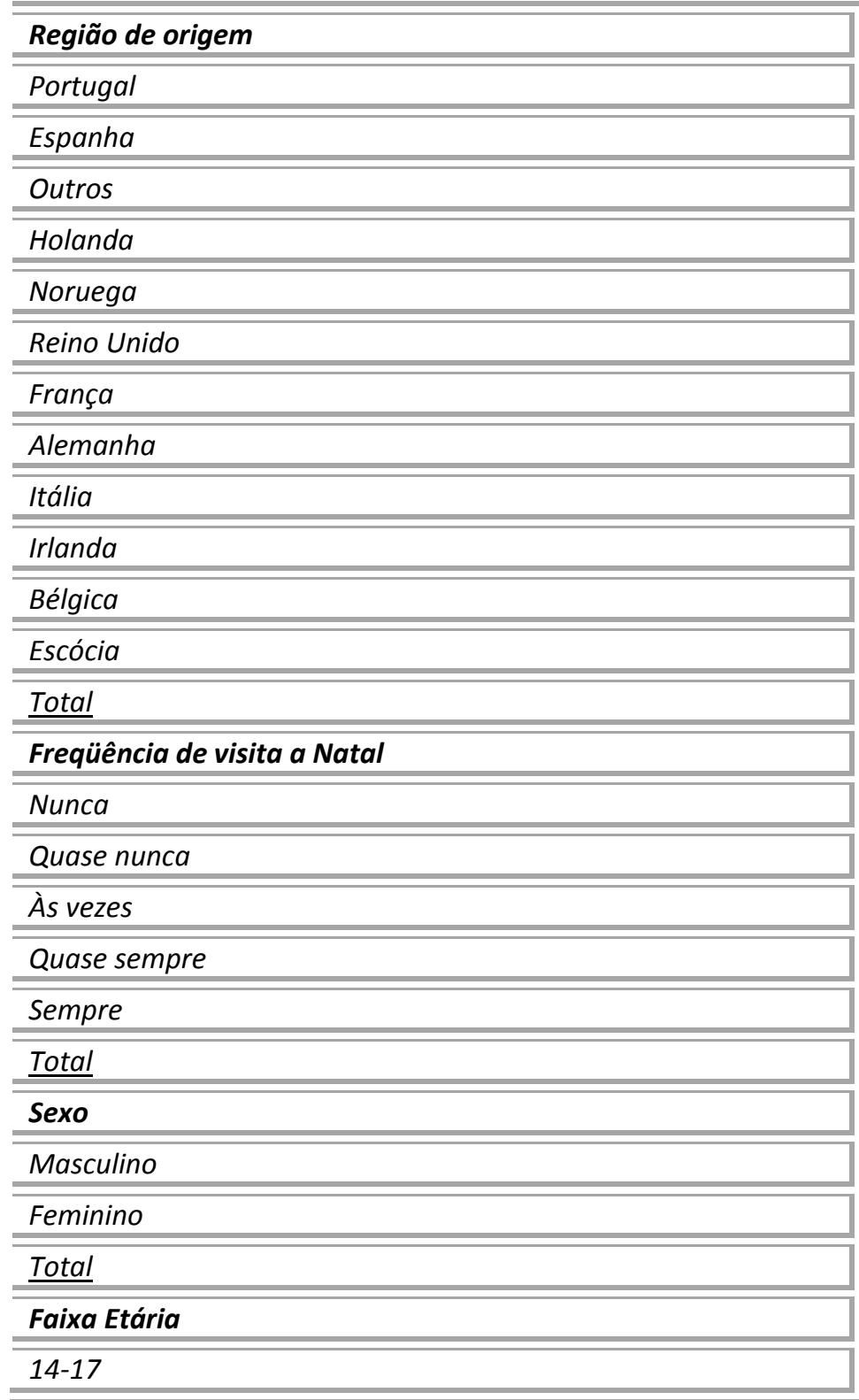

\begin{tabular}{|c|}
\hline Frequência \\
\hline 78 \\
\hline 31 \\
\hline 6 \\
\hline 43 \\
\hline 9 \\
\hline 39 \\
\hline 9 \\
\hline 11 \\
\hline 68 \\
\hline 1 \\
\hline 3 \\
\hline 2 \\
\hline 300 \\
\hline
\end{tabular}

10,4

Frequência

\begin{tabular}{|c|}
\hline \multicolumn{1}{|c|}{2,0} \\
\hline 14,5 \\
\hline 3,0 \\
\hline 13,1 \\
\hline 3,0 \\
\hline 3,7 \\
\hline 22,9 \\
\hline 0,3 \\
\hline 1,0 \\
\hline 0,7 \\
\hline 100 \\
\hline
\end{tabular}

\begin{tabular}{|c|}
\hline 87 \\
\hline 100 \\
\hline 83 \\
\hline 17 \\
\hline 5 \\
\hline$\underline{292}$ \\
\hline
\end{tabular}

Percentagem (\%)

\begin{tabular}{|c|}
\hline 29,8 \\
\hline 34,2 \\
\hline 28,4 \\
\hline 5,8 \\
\hline 1,7 \\
\hline$\underline{100}$ \\
\hline Percentagem (\%) \\
\hline 49 \\
\hline 51 \\
\hline 100 \\
\hline
\end{tabular}

Frequência

Percentagem (\%)

2,3 
Chagas, M. M., Sampaio, L. M. B., Santos, K. E. B. Análise da influência da imagem de destinos na satisfação e fidelidade a destinações de turismo de sol e praia: um estudo em Natal/RN

Tabela 01 - Perfil da amostra

(conclusão)

\begin{tabular}{|c|c|c|}
\hline Faixa Etária & Frequência & Percentagem (\%) \\
\hline $18-25$ & 51 & 17,1 \\
\hline $26-35$ & 81 & 27,2 \\
\hline $36-50$ & 96 & 32,2 \\
\hline $51-65$ & 47 & 15,8 \\
\hline Acima de 65 & 16 & 5,4 \\
\hline Total & 298 & 100 \\
\hline Escolaridade & Frequência & Percentagem (\%) \\
\hline Ensino fundamental & 14 & 4,9 \\
\hline Ensino médio & 67 & 23,6 \\
\hline Ensino superior & 155 & 54,6 \\
\hline Pós-graduação & 48 & 16,9 \\
\hline Total & $\underline{284}$ & $\underline{100}$ \\
\hline Vinda a Natal & Frequência & Percentagem (\%) \\
\hline Família & 119 & 42,7 \\
\hline Sozinho & 20 & 7,2 \\
\hline Amigos & 111 & 39,8 \\
\hline Grupos turísticos & 11 & 3,9 \\
\hline Outros & 18 & 6,5 \\
\hline$\underline{\text { Total }}$ & $\underline{279}$ & $\underline{100}$ \\
\hline Renda (Reais) & Frequência & Percentagem (\%) \\
\hline$R \$ 466,00$ a $R \$ 932,00$ & 11 & 6,1 \\
\hline$R \$ 933,00$ a $R \$ 1.866,00$ & 10 & 5,6 \\
\hline$R \$ 1.867,00$ a $R \$ 3.790,00$ & 50 & 27,8 \\
\hline$R \$ 3.791,00$ a $R \$ 7.582,00$ & 43 & 23,9 \\
\hline$R \$ 7.583,00$ a $R \$ 15.166,00$ & 24 & 13,3 \\
\hline Acima de $R \$ 15.166,00$ & 42 & 23,3 \\
\hline Total & $\underline{180}$ & $\underline{100}$ \\
\hline
\end{tabular}

Fonte: Dados do estudo.

A seguir é demonstrada a Análise Fatorial Exploratória (AFE) e a Análise de Regressão Linear Múltipla (RLM).

\subsection{Análise Fatorial Exploratória e Validação do Instrumento}

A Análise Fatorial Exploratória (AFE) foi utilizada a fim de identificar quais as dimensões da qualidade de serviços em destinos turísticos de Sol e Praia, de acordo com a percepção dos 
turistas internacionais, como também validar o instrumento desenvolvido para a investigação.

Para tanto, na Análise Fatorial se utilizou a Análise de Componentes Principais (ACP) como método para a extração de fatores, ao passo que o método de rotação selecionado foi o Varimax. Foram relacionadas apenas as variáveis com cargas fatoriais acima de 0,4, como também as dimensões cujo Eigenvalue obteve valores superiores a 1,0, ou seja, valores significativos para atestar a confiabilidade dos dados e resultados como afirma Corrar et al (2007).

Além disso, para confirmar a significância dos resultados foi realizado o teste KMO (Kaiser-MeyerOlkin), tendo atingido o valor de 0,922 como resultado, corroborado pelo Bartlett's Test of Sphericity com o valor de 7962,623, com significância zero (Sig, 0,000). Já o teste Alfa de Cronbach ( $\alpha$ ) foi aplicado em cada dimensão encontrada pela Análise Fatorial objetivando avaliar a consistência interna de cada uma. Nesse sentido, os valores obtidos que variaram entre 0,737 e 0,920, validaram a consistência interna dos fatores encontrados na AFE como assevera Hair Jro et al (2006).

Portanto, fica ratificada a qualidade e confiabilidade dos resultados. A Tabela 02 resume as principais informações auferidas na AFE, como também os testes e parâmetros que confirmam sua validade.

Tabela 02 - Dimensões da Qualidade de Serviços em Destinos de Sol e Praia

(continuação)

\begin{tabular}{|c|c|c|c|c|}
\hline Dimensões e variáveis & Fatorial & Eigenvalue & Variância (\%) & $(\alpha)$ \\
\hline 1 Praias & & 17,341 & 12,405 & 0,920 \\
\hline Limpeza da água e da areia & 0,553 & & & \\
\hline Segurança pública na praia & 0,586 & & & \\
\hline Equipamentos de apoio & 0,746 & & & \\
\hline Ambiente geral da praia & 0,722 & & & \\
\hline Outros serviços (Quiosques, duchas etc.) & 0,755 & & & \\
\hline Postos de vigilância e socorro & 0,648 & & & \\
\hline Equipamentos para prática de esportes & 0,714 & & & \\
\hline Placas indicadoras de risco, poluição e atividades náuticas & 0,640 & & & \\
\hline Demais freqüentadores & 0,738 & & & \\
\hline 2 Equipamentos Hoteleiros & & 3,279 & 11,449 & 0,905 \\
\hline Limpeza do hotel & 0,755 & & & \\
\hline Segurança do hotel & 0,804 & & & \\
\hline Qualidade ambiental do hotel & 0,785 & & & \\
\hline Equipamentos do quarto & 0,754 & & & \\
\hline Estética/Ambientação interna e externa ao hotel & 0,685 & & & \\
\hline Profissionalismo dos funcionários do hotel & 0,549 & & & \\
\hline Qualidade do restaurante do hotel & 0,503 & & & \\
\hline 3 Infra-Estrutura Pública & & 2,627 & 10,454 & 0,920 \\
\hline Comércio local/Shoppings & 0,459 & & & \\
\hline Limpeza e higiene em vias públicas & 0,544 & & & \\
\hline Sensação de segurança em vias públicas & 0,645 & & & \\
\hline Tranquilidade/Baixo nível de Ruídos & 0,729 & & & \\
\hline Tráfego/Congestionamento & 0,651 & & & \\
\hline Acesso aos atrativos turísticos & 0,658 & & & \\
\hline Equipamentos públicos (lixeira, praças etc.) & 0,605 & & & \\
\hline
\end{tabular}


Chagas, M. M., Sampaio, L. M. B., Santos, K. E. B. Análise da influência da imagem de destinos na satisfação e fidelidade a destinações de turismo de sol e praia: um estudo em Natal/RN

Tabela 02 - Dimensões da Qualidade de Serviços em Destinos de Sol e Praia

(conclusão)

Dimensões e variáveis Carga Fatorial Eigenvalue Variância (\%) $(\alpha)$

Conforto e Equipamentos do Aeroporto

0,525

\begin{tabular}{ll}
\hline 4 Restaurantes & \\
Limpeza do restaurante & 0,670 \\
Ambiente Geral & 0,747 \\
Qualidade da comida & 0,744 \\
Profissionalismo dos funcionários dos restaurantes & 0,602 \\
Localização e acesso & 0,672 \\
\hline 5 Serviços de Transportes, Informações e Financeiro & \\
Pontualidade dos vôos & 0,761 \\
Procedimentos de embarque e desembarque & 0,701 \\
Rede de transportes para saída do aeroporto & 0,695 \\
Postos de informações turísticas espalhados na cidade & 0,591 \\
Serviços financeiros, bancos & 0,480 \\
Táxis & 0,528
\end{tabular}

\section{Atrativos Turísticos}

$1,337 \quad 3,014 \quad 0,918$

$\begin{array}{ll}\text { Diversidade de atrativos e passeios } & 0,768\end{array}$

$\begin{array}{ll}\text { Passeios turísticos } & 0,777\end{array}$

$\begin{array}{ll}\text { Guia de turismo e bugueiro } & 0,741\end{array}$

$2,202 \quad 9,365 \quad 0,906$

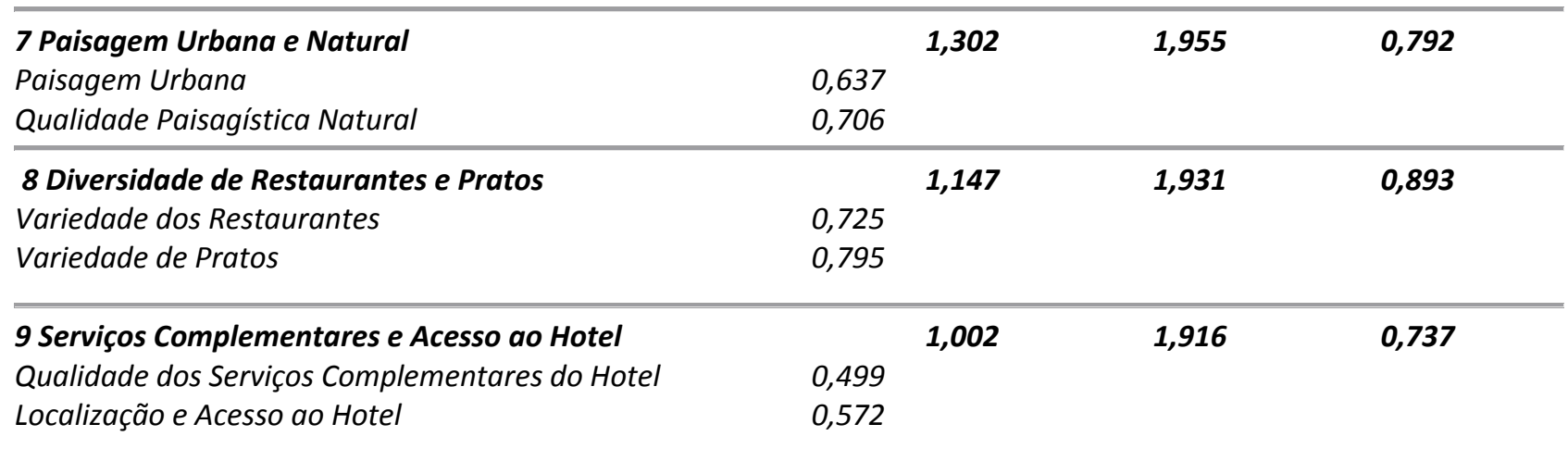

Total da Variância Explicada

77,807

Nota: A avaliação dos atributos variou entre os valores (01) e (10), respectivamente pior e melhor desempenho. Método de Extração: Análise de Componentes Principais.

Método de Rotação: Varimax.

KMO (Kaiser-Meyer-Olkin): 0,922.

Bartlett's Test of Sphericity: 7962,623 (Sig, 0,000).

Fonte: Dados do estudo.

De acordo com a Tabela 02, nove (09) são as dimensões da Qualidade de Serviços em destinações de Sol e Praia. A variância total explicada pelas dimensões atinge o valor de 77,807\%, ou seja, valor coerente com a literatura da área. Os fatores foram nomeados de acordo com as 
variáveis que as compuseram, da seguinte maneira: (1) Praias (Var.: 12,405\%), (2) Equipamentos Hoteleiros (Var.: 11,449\%), (3) Equipamentos Públicos (Var.: 10,454\%), (4) Restaurantes (Var.: 9,365\%), (5) Serviços de Transportes, Informações e Financeiro (Var.: 8,578\%), (6) Atrativos Turísticos (Var.: 3,014\%), (7) Paisagem Urbana e Natural (Var.: 1,955\%), (8) Diversidade de Restaurantes e Pratos (Var.: 1,931\%), e, por último, (9) Serviços Complementares e Acesso ao Hotel (Var.: 1,916\%), estando em consonância, as dimensões e variâncias explicadas, grosso modo, com os estudos de Chi \& Qu (2008), Hui, Wan \& Ho, (2007), Chen \& Tsai (2006), Rimmigton \& Kozak (2000), Andriotis, Agiomirgianakis \& Mihiotis, (2008), Yüksel (2001), Kozak (2000). Por fim, percebe-se que os três primeiros fatores concentram grande parte do percentual da variância explicada, sendo seguidos pelas dimensões Restaurantes e Serviços de Transportes, Informações e Financeiro. As dimensões da qualidade também podem ser observadas na Figura 02.

Figura 02 - Dimensões da Qualidade em Destinos de Sol e Praia

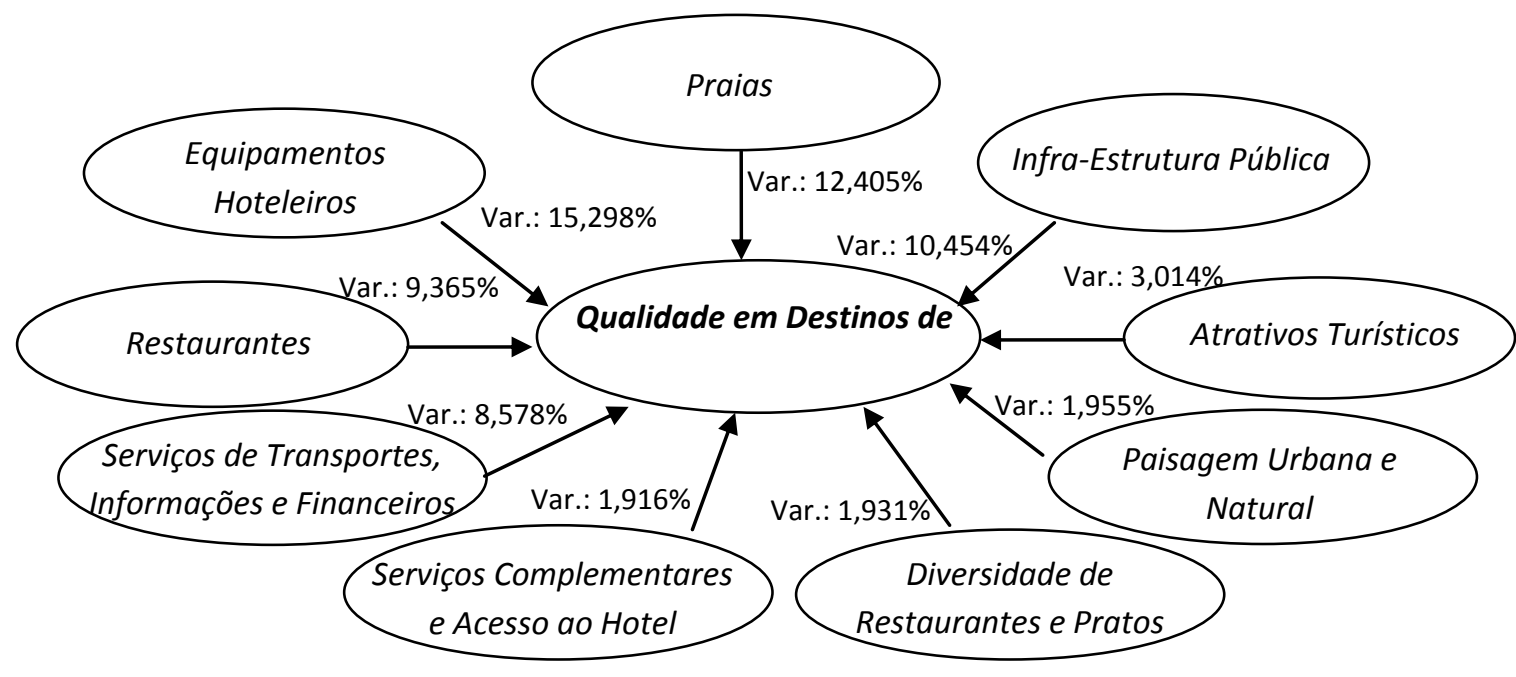

Fonte: Dados do estudo.

\subsection{Fatores influenciadores da Satisfação}

A análise das variáveis influenciadoras da satisfação com o destino foi desenvolvida a partir da Regressão Linear Múltipla (RLM). As variáveis independentes selecionadas na tentativa de explicação do comportamento da variável Satisfação (variável dependente) foram as nove (09) dimensões encontradas na Análise Fatorial Exploratória (AFE), a saber: (1) Praias, (2) Equipamentos Hoteleiros, (3) Infra-Estrutura Pública, (4) Restaurantes, (5) Serviços de Transportes, Informações e Financeiro, (6) Atrativos Turísticos, (7) Paisagem Urbana e Natural, (8) Diversidade de Restaurantes e Pratos, (9) Serviços Complementares e Acesso ao Hotel e a variável Imagem de Destinos. 
Com vistas a operacionalizar a análise por meio da RLM, o método Stepwise foi selecionado por proporcionar maior adequabilidade no tratamento do número elevado de variáveis do estudo, como também por proporcionar à análise controle com relação à multicolinearidade uma vez que esta é desestimulada a partir deste método. Além disso, como parâmetros para inclusão e exclusão de variáveis, por esse método, foi estipulado o valor de probabilidade $p$ igual ou menor a 0,05 , para inclusão, enquanto que para a exclusão foi igual ou superior a 0,10 (CORRAR et al, 2007).

De acordo com o teste F (F-ANOVA 174,156), com significância zero (Sig. 0,000), é possível afirmar que a hipótese do $\mathrm{R}^{2}$ ser igual a zero pode ser rejeitada. Conclui-se, de tal maneira, que as variáveis independentes possuem influência na variável dependente, confirmando, assim, a significância estatística do modelo encontrado (Wooldridge, 2010). O poder explicativo $\left(R^{2}\right)$ do modelo possui o valor de 0,784. Em outras palavras, o modelo encontrado para explicação da variável dependente Satisfação atingiu o poder explicativo de 78,4\%. A ausência de multicolinearidade também foi medida a partir dos testes VIF e Tolerance. Os resultados do primeiro se apresentaram inferiores a 10, considerado o limite para multicolinearidade inaceitável, ao passo que os relacionados ao segundo (Tolerance) obtiveram valores acima de 0,10 (Parâmetro máximo de multicolinearidade) como asseveram Corrar et al (2007).

A ausência de autocorrelação foi estimada através do teste Durbin-Watson, cujo resultado atingiu o valor de 1,659, ou seja, dentro dos parâmetros estipulados como adequados. $O$ teste $t$ de Student obteve, para cada variável, valor inferior a 0,05 corroborando com a afirmação da significância estatística de cada variável individualmente para a explicação da satisfação. A Tabela 03 resume os principais resultados, acompanhados dos testes estatísticos desenvolvidos para a validação do modelo.

Tabela 03 - Fatores Influenciadores da Satisfação em Destinos Turísticos de Sol e Praia

\begin{tabular}{lccccc}
\hline Variáveis independentes & 6 não padronizado & Erro padrão & B Padronizado & Teste t & Sig. \\
\hline $\begin{array}{lcccc}\text { (Constante) } \\
\text { Imagem }\end{array}$ & $-3,185$ & 0,171 & -- & $-18,678<0,0001$ \\
Serviços de Transportes, Informa- & 0,434 & 0,023 & 0,730 & $18,955<0,0001$ \\
ções e Financeiros & 0,136 & & & & \\
Restaurantes & 0,090 & 0,033 & 0,137 & $4,148<0,0001$ \\
Atrativos Turísticos & 0,077 & 0,030 & 0,090 & 2,989 & 0,0030 \\
Praias & 0,069 & 0,033 & 0,078 & 2,354 & 0,0190 \\
& & 0,031 & 0,070 & 2,256 & 0,0250 \\
\hline
\end{tabular}

Nota: $R^{2}=0,784 ; R^{2}$ Ajustado = 0,743; $F=174,156$ (Sig. 0,000); Durbin-Watson = 1,659.

Variáveis independentes $=$ Todas as listadas na tabela.

Variável dependente $=$ Satisfação.

Fonte: Dados do estudo

De acordo com a Tabela 03, observa-se que a Imagem de Destinos $(\beta 0,434)$ é a principal influenciadora da Satisfação, resultado em consonância com a literatura como pode ser visto em 
Chi \& Qu (2008), Echtner \& Ritchie (1991), Pike (2002), Gallarza, Gil \& Calderón (2002), Valls (1996) entre outros. Conclui-se, nesse sentido, que a Imagem exerce papel primordial e positivo no processo de satisfação do turista no destino, sendo então um direcionador que deve ser alvo de ações estratégicas.

A variável Serviços de Transporte, Informações e Financeiros $(\beta 0,136)$ se apresenta como a segunda mais influente, resultado decorrente, provavelmente, ao fato destes serviços serem considerados primordiais para um público cuja distância cognitiva e geográfica do destino receptor se revela elevada. Em outras palavras, como se trata de um público internacional, tais fatores encontram sua influência aumentada em decorrência das peculiaridades apresentadas por esse público, como, por exemplo, dificuldades com o idioma e diferenças culturais entre outras, que dificultam, sobremaneira, o acesso a serviços básicos responsáveis pela sensação de segurança no destino, como o deslocamento, o acesso as informações sobre o que o destino oferece que, por sua vez, impactará fortemente sua percepção de qualidade e satisfação com o portfólio oferecido pela destinação, entre outros serviços oferecidos.

Por último, percebe-se que o portfólio de atrativos do destino, ou seja, aspectos relacionados a praias, gastronomia e outros atrativos são as três outras dimensões que completam o modelo. Dito de outro modo, as dimensões Restaurantes $(\beta 0,090)$, Atrativos Turísticos $(\beta 0,077)$ e Praias $(\beta$ 0,069), em conjunto, demonstram a grande influência, para o público internacional, exercida por esses fatores relacionados ao potencial atrativo do destino de férias, fato coerente com a literatura uma vez que estes são os fatores estímulo para a escolha da destinação de férias, como também via de regra o motivo principal do deslocamento para outro lugar fora de seu estorno habitual (Yoon; Uysal, 2005).

\subsection{Fatores influenciadores da Fidelidade}

Para fins de avaliação dos fatores influenciadores da Fidelização também foi utilizada a Análise de Regressão Linear Múltipla (RLM), de acordo com os mesmos parâmetros estabelecidos na análise anterior. A variável dependente Fidelização representa um fator originado de duas outras variáveis, ou seja, (1) pretensão de retornar ao destino e (2) propensão a recomendar o destino. As variáveis explicativas, por sua vez, foram constituídas pelas dimensões da Qualidade de Serviços, junto a Satisfação e a Imagem de Destinos. O quadro resumo do modelo pode ser observado na Tabela 04. 
Tabela 04 - Fatores Influenciadores da Fidelidade aos Destinos Turísticos de Sol e Praia

Variáveis independentes B não padronizado Erro padrão B Padronizado Teste $t$ Sig.

\begin{tabular}{|c|c|c|c|c|c|}
\hline (Constante) & $-1,990$ & 0,251 & --- & $-7,942$ & $<0,0001$ \\
\hline Imagem & 0,271 & 0,034 & 0,455 & 7,998 & $<0,0001$ \\
\hline Satisfação & 0,418 & 0,057 & 0,417 & 7,279 & $<0,0001$ \\
\hline \multicolumn{6}{|c|}{ Diversidade de Restaurantes e } \\
\hline Pratos & 0,103 & 0,030 & 0,104 & 3,460 & 0,0010 \\
\hline \multicolumn{6}{|c|}{ Serviços Complementares e Acesso } \\
\hline ao Hotel & 0,088 & 0,030 & 0,089 & 2,952 & 0,0030 \\
\hline Restaurantes & $-0,061$ & 0,030 & $-0,062$ & $-2,012$ & 0,0450 \\
\hline
\end{tabular}

Nota: $R^{2}=0,793 ; R^{2}$ Ajustado = 0,734; $F=166,290$ (Sig. 0,000); Durbin-Watson $=1,400$.

Variáveis independentes $=$ Todas as listadas na tabela.

Variável dependente $=$ Fidelidade.

Fonte: Dados do estudo.

Do exposto na Tabela 04, observa-se que os dois fatores que exercem maior influência junto a Fidelização do turista são a Imagem e a Satisfação. Nesse sentido, fica comprovada a relação da Imagem ( $\beta$ 0,271 ) como um dos principais direcionadores da Fidelização como é amplamente reconhecido na literatura Bosque \& Martín (2008), Chen \& Tsai (2006), Chi \& Qu (2008), Echtner \& Ritchie (1991), Pike (2002), Gallarza, Gil \& Calderón (2002) e Valls (1996). Além disso, a própria relação entre Satisfação ( $\beta$ 0,418 ) e Fidelidade também é bastante contemplada nos estudos da área (Bosque \& Martín, 2008; Chen \& Tsai, 2006; Zabkar; Brencic \& Dmitrovic, 2009; Yoon \& Uysal, 2005; Correia \& Pimpão, 2005). Outro fator importante, foi a Diversidade de Restaurante e Pratos $(\beta 0,103)$, seguidas das demais com baixa influência. A Figura 03 demonstra o modelo final encontrado no estudo.

Figura 03 - Modelo encontrado pelo estudo.

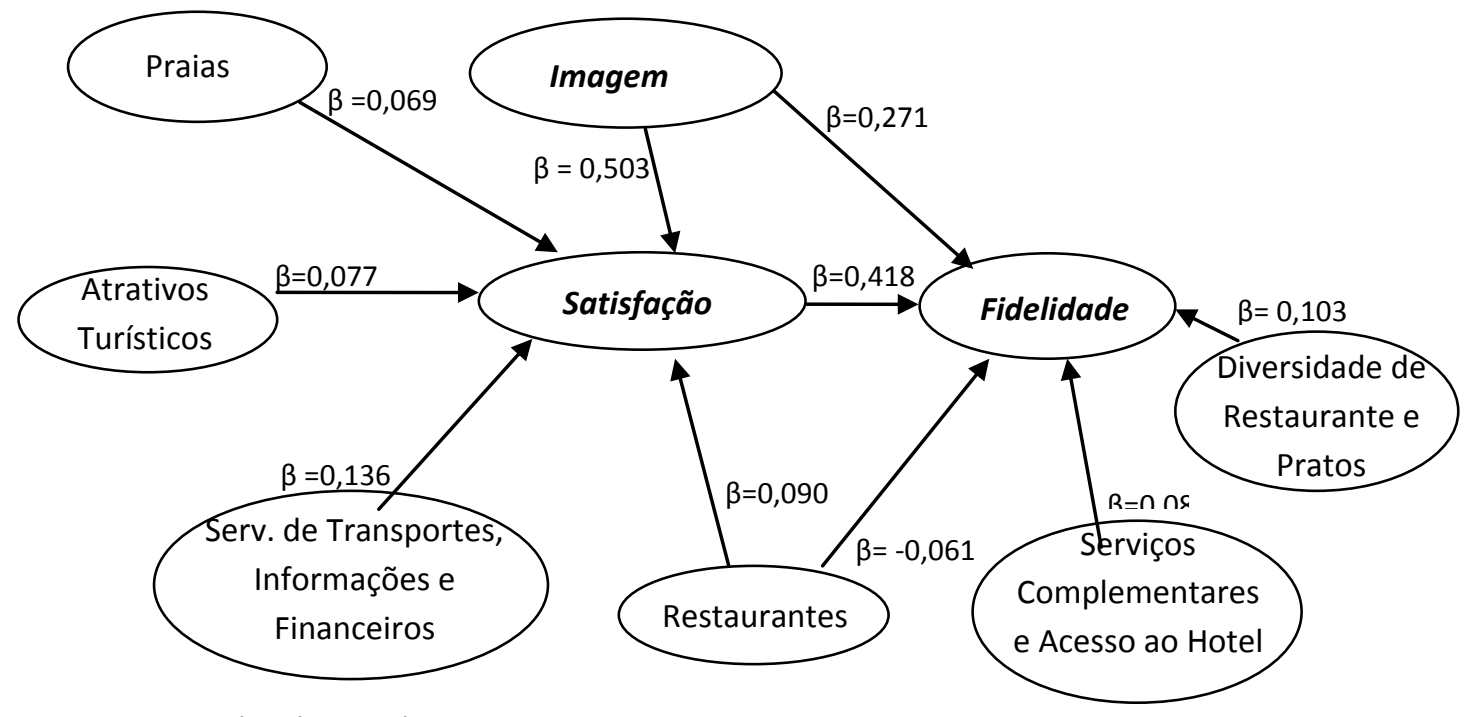

Fonte: Dados do estudo. 


\section{CONCLUSÃO}

O estudo desenvolvido, do ponto de vista teórico, contribui para diminuição da lacuna encontrada com relação à investigação da relação entre os constructos citados e ao desenvolvimento de instrumentos próprios de avaliação da qualidade, satisfação e fidelidade no setor de turismo. Do ponto de vista prático, as implicações gerenciais do estudo versam sobre o levantamento de dados essenciais sobre o comportamento do consumidor em destinos de Sol e Praia, especialmente do caso de Natal/RN, o que, por sua vez, pode ser utilizado como fonte de informações para o desenvolvimento de estratégias mais eficientes e eficazes no cumprimento dos objetivos de aumento de competitividade e sustentabilidade, considerada um imperativo atualmente, em destinações turísticas desse segmento, tais como Natal e outras cidades do nordeste.

Dentre os principais resultados, cabe ressaltar que as dimensões da qualidade dos serviços em destinos de Sol e Praia encontradas foram nove (09), a saber: (1) Praias, (2) Equipamentos Hoteleiros, (3) Equipamentos Públicos, (4) Restaurantes, (5) Serviços de Transportes, Informações e Financeiro, (6) Atrativos Turísticos, (7) Paisagem Urbana e Natural, (8) Diversidade de Restaurantes e Pratos e (9) Serviços Complementares e Acesso ao Hotel.

Além disso, conclui-se também que a Imagem de Destinos é o principal fator influenciador da Satisfação do turista, relação amplamente corroborada pela literatura da área, seguida pelos fatores Serviços de Transporte, Informações e Financeiros, Restaurantes, Atrativos Turísticos e Praias. A Fidelidade, por sua vez, é influenciada, principalmente, pelas dimensões Satisfação e Imagem do Destino como encontrados em outros estudos da área. Portanto, para fins práticos, cabe aos destinos de Sol e Praia, em especial o estudado, investir em aspectos que colaborem com a melhoria de sua Imagem junto ao seu público consumidor, os turistas, uma vez que esta tem influência direta, forte e positiva junto à satisfação e a fidelidade ao destino.

Como limitação, a investigação apresenta um relato da percepção de um público específico (o internacional) de uma destinação de Sol e Praia particular (Natal/RN) em apenas uma temporada do ano, não demonstrando, dessa maneira, a percepção do público nacional frequentador da cidade, nem tampouco a percepção de turistas que viajam em outros períodos do ano. Além disso, não foi contemplada a percepção de turistas em visita a outros destinos com formatação comercial similar.

Para futuros estudos, recomenda-se a replicação desse tipo de investigação em outros contextos e públicos a fim de se observar se os resultados permanecem apontando as mesmas tendências de comportamento. Faz-se interessante também o uso de técnicas tais como análise fatorial confirmatória e análise de equações estruturais com vistas a realizar uma nova confirmação da relação causal entre as dimensões trabalhadas, como também inserir outras dimensões nas análises que possam interferir na satisfação e fidelidade tais como o apego e a 
Chagas, M. M., Sampaio, L. M. B., Santos, K. E. B. Análise da influência da imagem de destinos na satisfação e fidelidade a destinações de turismo de sol e praia: um estudo em Natal/RN

emoção entre outros, que a exemplo da imagem de destinos apela para a relação afetiva do turista com local de férias.

\section{REFERÊNCIAS}

AKBABA, A (2006). Measuring service quality in the hotel industry: A study in a business hotel in Turkey. Hospitality Management. 25, 170-192.

ALBACETE-SÁEZ, C. A.; FUENTES-FUENTES, M. M; LLORÉNS-MONTES; F. J (2007). Service quality measurement in rural accommodation. Annals of Tourism Research, 34(1), 45-65.

ANDRIOTIS, K. et al (2008). Measuring tourist satisfaction: A factor-cluster segmentation approach. Journal of Vacation Marketing, 14(3), 221-235.

ARMSTRONG, R. W.; MOK, C.; GO, F. M. (1997). The importance of cross-cultural expectations in the measurement of service quality perceptions in the hotel industry. International Journal of Hospitality Management, 16(2), 181-190.

ASSAEL, H. (1999). Comportamiento del consumidor. México: International Thompson Editores.

ANDERECK, K. L et al. (2000). Residents' perceptions of community tourism impacts. Annals of Tourism Research, 32(4), 1056-1076.

BAKER, D. A.; CROMPTON, J. L. (2000). Quality, Satisfaction and Behavioral Intention. Annals of Tourism Research, 27(3), 785-804.

BEDIA, A. M. S.; FERNÁNDEZ, M. C. L.; LÓPEZ, R. G. (2007). Gestión de calidad y turismo: revisión e implicaciones para futuras investigaciones. Cuadernos de Turismo, 20, 251-266.

BOSQUE, I. R.; MARTíN, H. S. (2008). Tourist satisfaction: A cognitive-affective model. Annals of Tourism Research, 35(2), 551-573,.

BALOGLU, S.; MCCLEARY, K. W. (1999). A model of destination image formation. Annals of Tourism Research, 26(4), 868-897.

BEERLI, A.; MARTíN, J. D. (2004a). Tourists' characteristics and the perceived image of tour destinations: a quantitative analysis-a case study of Lanzarote, Spain. Tourism Management, 25, 623-636. (2004b). Factors influencing destination image. Annals of Tourism Research, 31(3), 657-681.

CHEN, C. F.; TSAI, D. C. (2007). How destination image and evaluative factors affect behavioral intentions?. Tourism Management, 28, 1115-1122.

CHI, C. G. Q.; QU, H. (2008). Examining the structural relationships of destination image, tourist satisfaction and destination loyalty: An integrated approach. Tourism Management, 29, 624-638.

CORREIA, A.; PIMPÃO, A. (2008). Decision-making processes of Portuguese tourist travelling to South America and Africa. International Journal of Culture, Tourism and Hospitality Research, 2(4), 330-373.

CRONIN, J.; TAYLOR, S. (1994). SERVPERF versus SERVQUAL: reconciling performance based and perceptions minus expectations measurement of service quality. Journal of Marketing, 58(1), 125-131.

CORRAR, L. J.; PAULO, E.; DIAS FILHO, J. M. (2007). Análise multivariada: Para cursos de administração, ciências contábeis e economia. São Paulo: Atlas.

ECHTNER, C. M.; RITCHIE, J. R. B. (1991). The meaning and measurement of destination image. The Journal 
of Tourism Studies, 14 (1), 37-47.

GALLARZA, M. G; CALDERÓN, H. G.; GIL, I. S. (2002). Destination image: towards a conceptual framework. Annals of Tourism Research, 29 (1), 56-78.

GUTIÉRREZ, H. S. M. (2005). Estudio de La imagen de destino turístico y el processo global de satisfacción: adopción de un enfoque integrador. Santander (ES). Tese Departamento de Administracion de Empresas de La Universidad de Cantabria.

HAIR, J. F. Jr. et al. (2006). Análise multivariada de dados. São Paulo: Bookman.

HUI, T. K.; WAN, D.; HO, A. (2007). Tourists' satisfaction, recommendation and revisiting Singapore. Tourism Managemen,. 28, 965-975.

JUWAHEER, T. D. (2004). Exploring international tourists' perceptions of hotel operations by using a modified SERVQUAL approach - a case study of Mauritius. Managing Service Quality, 14 (5), 350-364.

JOHNSON, M. D. et al. (2001). The evolution and future of national customer satisfaction index models. Journal of Economic Psychology, 22, 217-245.

KOZAK, M. (2001). Comparative assessment of tourist satisfaction with destinations across two nationalities. Tourism Management, 22, 391-401.

MENG, F.; TEPANON, Y.; UYSAL, M. (2005). Measuring tourist satisfaction by attribute and motivation: The case of a nature-based resort. Journal of Vacation Marketing, 14 (1), 41-56.

MOWFORTH, M.; MUNT, I. (2006). Tourism and sustainability: development and new tourism in the third world. New York: Routledge.

NICOLAU, J. L.; MÁS, F. J. (2006). The influence of distance and prices on the choice of tourist destinations: The moderating role of motivations. Tourism Management, 27, 982-996.

NEAL, J. D.; GURSOY, D. (2008). A Multifaceted Analysis of Tourism Satisfaction. Journal of Travel Research. $47(1), 52-63$.

OLIVER, R. L. (1993). Cognitive, affective, and attribute bases of the satisfaction response. Journal of Consumer Research, 20, 418-430.

OMT. (2005). Introdução a metodologia da pesquisa em turismo. São Paulo: Roca.

PIKE, S. (2002). Destination image: analysis a review of 142 papers from 1973 to 2000. Tourism Management, 23(05), 541-549.

PARASURAMAN, A.; V. ZEITHAML; L. BERRY. (1985). A conceptual model of service quality and its implication for future research. Journal of Marketing, 49, p. 41-50.

. (1988). Servqual: A multiple-item scale for measuring consumer perceptions of service quality. Journal of Retailing, 64 (1), 12-40.

RITCHIE, J.R. B.; CROUCH, G. I. (2003). The competitive destination: A sustainability perspective. Canadá: University of Calgary.

RIMMINGTON; M.; KOZAK, M. (2000). Tourist Satisfaction with Mallorca, Spain, as an Off-Season Holiday Destination. Journal of Travel Research. 38, 260-269.

SIRAKAYA, E.; WOODSIDE, A. G. (2005). Building and testing theories of decision making by travelers. Tourism Management, 26, 815-832.

SECTUR/RN (2007). Planejamento estratégico SECTUR 2004-2007. Web site. Acedido em dezembro 31, 
2007, em www.natal.rn.gov.br/.

UM, S.; CHON, K.; RO, Y. (2006). Antecedents of revisit intention. Annals of Tourism Research, 33 (4), 11411158.

VALLS, J. F. (1996). Las claves del mercado turístico: cómo competir en el nuevo entorno. Bilbao: Deusto.

WTTC. (2007). Executive summary: travel \& tourism climbing to new heights: the 2006 travel \& tourism economic research. Web site. Acedido em fevereiro 24, 2007, em http://www.wttc.org/.

WANG, X.; ZHANG, J.; GU, C.; ZHEN, F. (2009). Examining Antecedents and Consequences of Tourist Satisfaction: A structural modeling approach. Tsinghua Science and Technology, 14 (3), 397-406.

WOOLDRIDGE, J. M. (2010). Introdução à econometria: uma abordagem moderna. (4a ed.). São Paulo: Cegage Learning.

YILMAZ, I. (2009). Measurement of service quality in the hotel industry. Anatolia: An International Journal of Tourism and Hospitality Research, 20 (2), 375-386.

YÜKSEL, A. (2001). Managing customer satisfaction and retention: A case of Turkey tourist destinations. Journal of Vacation Marketing, 7 (2), 153-168.

YOON, Y.; UYSAL, M. (2005). An examination of the effects of motivation and satisfaction on destination loyalty: a structural model. Tourism Management, 26, 45- 56.

ZABKAR, V.; BRENCIC, M. M.; DMITROVIC, T. (2009). Modelling perceived quality, visitor satisfaction and behavioural intentions at the destination level. Tourism Management, 31 (4), 537-546, 2009.

Artigo recebido em: 26/07/2013.

Artigo aprovado em: 19/08/2013. 